\title{
Evocación de memorias a través del cine de oro mexicano
}

\author{
Annika Maya Rivero* \\ Universidad Autónoma del Estado de México, Facultad de Arquitectura y Diseño \\ Toluca, Estado de México, México \\ Maricruz Castro Ricalde ${ }^{* *}$
}

Instituto Tecnológico de Estudios Superiores de Monterrey, Departamento de Comunicación y Humanidades Toluca, Estado de México, México

Recibido: 23 de agosto 2017; aceptado: 7 de septiembre 2017

\begin{abstract}
Resumen
La terapia de reminiscencia, la cual consiste en la evocación de memorias en personas con demencia a través de materiales de soporte, es un recurso poco utilizado en países latinoamericanos, debido a la carencia de materiales apropiados y a la falta de una guía o método fácil de ejecutar. El uso del cine como herramienta de la terapia de reminiscencia ha sido una incursión reciente, su aplicación se ha llevado a cabo en el Reino Unido así como en los Estados Unidos de Norteamérica. Bajo el supuesto: El cine puede detonar recuerdos en las personas mayores con demencia, además de funcionar como integrador de diversos elementos de la cultura material simbólica referentes al tiempo correspondiente al llamado golpe de reminiscencia, se realizaron tres intervenciones en un grupo de 15 personas mayores con demencia, ubicado en la ciudad de Toluca, México. Se proyectó la película del cine mexicano de la época de oro: "Yo soy gallo dónde quiera (Jimmy)" (1954). Su proyección fue dividida en tres sesiones, en las cuales se utilizó material de soporte, extraído de la película, dicho material fue clasificado de acuerdo a los sentidos del cuerpo humano con el fin de promover la estimulación sensorial en los participantes. Las personas mayores con demencia se mostraron interesadas al hablar de películas y cine. Se obtuvieron recuerdos autobiográficos de los participantes, así como cambios positivos en su estado de ánimo y conducta.
\end{abstract}

Palabras clave: Demencia | cine mexicano | terapia de reminiscencia | adultos mayores mexicanos | memoria

Evocation of memories through Mexican gold cinema

\begin{abstract}
Reminiscence therapy, which consists of memory recall in people with dementia through support materials, is a resource rarely used in Latin American countries, due to the lack of appropriate materials and the lack of a guide or method. The use of cinema as a tool of reminiscence therapy has been a recent incursion, its application has been carried out in the United Kingdom as well as in the United States of North America. Under the assumption: Cinema can detonate memories in elderly people with dementia, besides functioning as an integrator of various elements of the symbolic material culture referring to the time corresponding to the so-called reminiscence bump, three interventions were performed in a group of 15 elderly people with dementia, located in the city of Toluca, Mexico. The film of the Mexican cinema of the golden era was projected: "I am rooster where I want (Jimmy)" (1954). Its projection was divided in three sessions, in which material of support was used, extracted of the film, this material was classified according to the human senses in order to promote the sensorial stimulation in the participants. Older people with dementia are interested in talking about movies and movies. There were autobiographical memories of the participants, as well as positive changes in mood and behavior.
\end{abstract}

Key words Dementia | Mexican cinema |Reminiscence therapy | Mexican elderly | Memory

\section{Antecedentes}

La demencia es, quizá, la enfermedad que ocupará a muchos investigadores, y a la sociedad en general, durante el siglo XXI. Se sabe que al día de hoy esta ha sido declarada por la Organización Mundial de la Salud (OMS) como una prioridad de salud pública (OMS, 2015).
Los mayores avances en cuanto a desarrollo de modelos de atención centrados en la persona los han llevado a cabo países desarrollados. Un ejemplo son las villas para la demencia ubicadas en Hogeweyk, Amsterdam, estas tienen áreas que dependen del ambiente en el cual se desarrolló la persona a lo largo de su vida. La división de las áreas se hace por medio de

* maricruz.castro@itesm.mx

**annikamr@gmail.com 
aspectos socio culturales (Dementia Village Architects, 2015).

En varios países se han desarrollado distintas estrategias para tratar la demencia. Escocia ha desarrollado un plan nacional el cual es citado por la OMS dentro del reciente informe sobre envejecimiento y salud (2015). Dicho plan busca que las personas con demencia vivan en sus hogares por más tiempo con buena calidad de vida y que las comunidades estén abiertas a las personas con este padecimiento, entre otros objetivos.

Pero ¿qué pasa cuando se habla del tema en América Latina? La historia es distinta, mientras que en los países desarrollados se planea que las comunidades enteras sean amigables con las personas con demencia y sus familias. En lugares como México, aún se sigue luchando con la regulación de centros de día y temas que rebasan el avance de la enfermedad.

De acuerdo con el reporte de Alzheimer's Disease International (Organización internacional de la enfermedad de Alzheimer), eran los países en vías de desarrollo, quienes, en el año 2015 presentaban mayor incidencia con respecto a este padecimiento, además son estos países de bajo y medio ingreso quienes presentarán un incremento más rápido de casos en los próximos años (Prince et al., 2015).

Debido a la escasez de recursos que van desde económicos hasta profesionales, la implementación de estrategias utilizadas en países desarrollados es inviable en la mayoría de los países del mundo que son similares a México. En este país sólo existen 400 médicos geriatras para atender a la población envejecida (Aguirre, 2015), población que ronda los 10 millones de personas (INEGI, 2010), además se estima que 800,000 personas padecen algún tipo de demencia (Sosa, 2016). La generación de miles de geriatras o especialistas tardará, así que la aplicación y conocimiento de estrategias fáciles de aplicar por cuidadores informales es imperativa.

Aunado a los retos económicos y profesionales que se presentan en México y Latinoamérica, el uso de material infantilizante dentro de las terapias para adultos mayores, con o sin demencia, es una constante que puede observarse en los diversos centros de día a lo largo del país. La experta en la atención centrada en la persona con demencia, Teresa Martínez Rodríguez, promueve no infantilizar las terapias para adultos mayores (2011:156).

Existen intervenciones psicosociales que buscan mejorar la calidad de vida de las personas con demencia, al tiempo que hacen viable cierto tipo de terapias. La terapia de reminiscencia (TR) es una de estas, se uti- lizan: Álbumes de fotos, objetos históricos y significantes, libros de historia de vida, cajas de memoria o kits de reminiscencia, entre otros (Cotelli et al., 2012; Latha et al., 2014).

Existe evidencia de que la TR puede mejorar el estado de ánimo así como algunas actividades cognitivas, reducir la depresión e incrementar las ondas cerebrales que mejoran las funciones afectivas (Cotelli et al., 2012; Kalu, 2015) además de que la TR puede mejorar la memoria autobiográfica (Cotelli et al., 2012).

La terapia de reminiscencia tiene un soporte teórico en distintos modelos de memoria como el de Endel Tulving (1972). La memoria autobiográfica está conformada por una serie de memorias cruzadas y codificadas, cada recuerdo autobiográfico se relaciona con el yo del momento que funcionaba cuando el recuerdo se formó, cuando una parte del conocimiento autobiográfico se activa por un detonante entonces se puede desencadenar una serie de recuerdos (Williams y Conway, 2009).

Dentro de la memoria autobiográfica existe una teoría sobre el golpe de reminiscencia, que fue descubierto en 1986 por Rubin, Wetzler y Nebes (Berntsen y Bohn, 2009) y consiste en una larga lista de eventos positivos ocurridos en nuestra vida, situaciones en las cuales se lograron objetivos incluidos en nuestro guion de vida (Williams y Conway, 2009). El periodo del golpe de reminiscencia comprende desde los 10 hasta los 30 años de edad del ser humano, el mayor número de recuerdos evocan a los 20 años de edad (Williams y Conway, 2009). Además de los recuerdos personales o privados, existen los recuerdos flash, los cuales se refieren a los recuerdos impactantes a consecuencia de eventos internacionales o nacionales. Estos, se relacionan mucho con la cultura y la sociedad, estas memorias definen la identidad de cada generación (Williams y Conway, 2009).

Tanto el golpe de reminiscencia, como los recuerdos flash son elementos que deben de estar presentes en la aplicación de la TR. Pese a que aún para muchos especialistas, la terapia de reminiscencia carezca de elementos metodológicos que le otorguen una validación (Cotelli et al., 2012), es indudable el hecho de que los seres y los objetos están ligados, y que los objetos cobran esta complicidad y valor afectivo (Baudrillard, 2010).

La relación que nuestros procesos cognitivos mantienen con los objetos que nos rodean ha sido tal, que medimos nuestra evolución humana con base en los elementos físicos que desarrollamos (Malafouris y Renfrew, 2013). Así que la relación que como seres humanos mantenemos con el objeto externo es tan íntima que, para ciertos 
autores, es en esta interacción en donde se encuentra la evolución cognitiva (Malafouris y Renfrew, 2013).

El uso del cine como herramienta de la TR ha sido una incursión reciente. Ejemplos de su aplicación son: El proyecto: Banco de recuerdos en el Reino Unido y el proyecto: Nos vemos en las películas... y recordemos, en los Estados Unidos de Norte América.

El Banco de recuerdos, fue llevado a cabo por el Archivo Cinematográfico de Yorkshire y un panel de especialistas en demencia compuesto por personas provenientes de la Sociedad de Alzheimer del Reino Unido y los hogares metodistas para los mayores. Este proyecto consistió en seleccionar películas familiares filmadas entre 1940 y 1970 con la finalidad de revivir el pasado para estimular a los pacientes a recordar (BBC, 2012). Las cintas se centran en actividades de la vida diaria, ahora pueden adquirirse en paquetes en formato DVD además de una guía y recursos para la aplicación exitosa de las sesiones de reminiscencia (Memory bank, 2012).

En lo que respecta al proyecto: Nos vemos en las películas... y recordemos, este hace uso de pequeños cortos de películas clásicas, seguido de una discusión guiada por el moderador. De acuerdo con la fundación que soporta el proyecto:

Este programa demuestra como las películas pueden ser una forma de tratamiento para algunos de los síntomas asociados con el Alzheimer y algunas demencias. El cine tiene el poder de conectarnos con recuerdos emocionales profundamente arraigados, de la clase que nunca nos deja (Im still here foundation, 2016).

\section{Desarrollo}

Bajo el supuesto: El cine puede detonar recuerdos en las personas mayores con demencia, además de funcionar como integrador de diversos elementos de la cultura material simbólica referentes al tiempo correspondiente al llamado golpe de reminiscencia, es que se planteó la realización de la intervención que se explica en el presente documento.

El objetivo fue hacer uso de elementos sensoriales evocadores de recuerdos basados en el cine mexicano de la edad de oro y generar así una terapia de reminiscencia viable en su ejecución.

Se encontraron diversas problemáticas para la realización de la investigación: La falta de validación de la TR, sobre todo al hablar de qué estímulos funcionan, cómo aplicarlos y a quién; fue entonces que bajo la perspectiva interpretativa del interaccionismo simbólico se planteó iniciar un proceso de análisis sobre la vinculación entre la persona y su ambiente, pues al hablar de memoria estamos hablando de un registro sensorial (Dulcey-Ruiz, 2015).

Jean Baudrillard sostiene que, los objetos no son sólo un cuerpo material, sino un recinto mental en el cual yo reino, yo soy el sentido (2010). El principal respaldo para la historia no es la filosofía, sino el arte, que sirve para desarrollar y modificar la imagen que un grupo tiene de sí mismo (Geertz, 2006), en esto y en el hecho de que los recuerdos autobiográficos localizan al ser humano en un tiempo socio-histórico y casi siempre incluyen aspectos visuales (Williams y Conway, 2009) se sustenta el uso del cine como una herramienta para la terapia de reminiscencia.

Al presenciar una película surge un proceso de identificación (García-Riera, 1974) y este proceso es otra característica pertinente a ser resaltado para utilizar al cine como herramienta en la TR. En la terapia de reminiscencia los sujetos interpretan sus experiencias al tiempo que pueden ser alterados por éstas (Iacub, 2011) y el cine mexicano de la época de oro resultó, de acuerdo con las observaciones no participantes, una excelente vía para la re-interpretación de las experiencias personales de los hoy adultos mayores.

La segunda problemática fue la gran variedad de películas que pueden proyectarse como parte de una terapia del recuerdo, cuales escoger y por qué así como la metodología de la presentación fueron situaciones que debieron de solucionarse. Se encontró que el cine mexicano de la "Edad de Oro" tuvo un papel preponderante en la formación de una cultura común [...] hecho que rebasó las salas cinematográficas mexicanas (Castro-Ricalde y Irwin, 2011).

La etapa del cine de oro mexicano puede acotarse durante los años de 1936 a 1956 (Castro-Ricalde, 2014). Por ello el cine mexicano de la época dorada puede fungir como un eje para la terapia de reminiscencia. Al tomar en cuenta que, al día de hoy, las personas que tienen entre 80 y 89 años de edad, tanto en el continente americano como en el europeo son quienes más incidencia de demencia presentan (Prince et al., 2015) se puede relacionar que son personas que a sus veintes (golpe de reminiscencia) presenciaron al cine mexicano de la época de oro.

Entonces, el cine mexicano se pondera como un eje viable por el hecho de coincidir no sólo temporalmente, sino culturalmente con las personas a quienes hoy aqueja la enfermedad que nos ocupa, personas no sólo mexica- 
nas, sino argentinas, colombianas, cubanas, venezolanas, estadounidenses, españolas, etcétera (Castro-Ricalde y Irwin, 2011).

\section{Método}

Haciendo uso del método cualitativo, se realizó un muestreo no probabilístico discrecional escogiendo a un grupo de personas adultas mayores pertenecientes a una residencia geriátrica especializada en personas con demencia, ubicada en la ciudad de Toluca, Estado de México, México. El grupo se conforma por 10 mujeres y 5 hombres, la cantidad de personas varía pues además de ser una residencia es también centro de día. El grupo de personas fue escogido por cumplir con las siguientes características: La mayoría presenta algún tipo de demencia y la edad promedio de grupo rondaba los 84 años.

Durante 10 meses se llevó a cabo una investigación de carácter etnográfico, se hicieron visitas semanales de una hora, con el objetivo de conocer a las personas de la residencia geriátrica. Poco a poco se fue participando más en las actividades hasta el punto de poder interactuar con cada una de ellas y corroborar que, tal como la literatura indica, los adultos mayores con demencia mantienen una vinculación profunda con la cultura material (C. Buse y Twigg, 2014; Christina Buse y Twigg, 2015).

Se realizó investigación en el archivo municipal de Toluca, México con la finalidad de conocer qué películas mexicanas fueron las más taquilleras durante un periodo de tiempo perteneciente al golpe de reminiscencia de las personas mayores de la residencia geriátrica.

Debido a la amplia información disponible, se acotó la búsqueda al mes de marzo de 1954, esto puesto que la mayor cantidad de información existente, que podía cruzarse con la encontrada en el periódico más popular de la región, correspondía a este mes. Se accedió a auditorías que se realizaron a los cines de la ciudad de Toluca, en donde se exhibían los ingresos totales de los cines por cada día del mes de marzo. Se cruzó la información con la cartelera exhibida en los días de la auditoría para así encontrar las películas más taquilleras durante ese lapso de tiempo. Fueron encontradas dos películas. Posteriormente, se escogió una de ellas para iniciar su aplicación, un factor importante para haber seleccionado las películas fue el cine en el cual fueron exhibidas, en este caso se eligió el más popular de la época. La película encontrada lleva por nombre Yo soy gallo donde quiera (1954).

Se observó y analizó la película en busca de elementos sensoriales que pudieran enriquecer la intervención, esto con base en la aplicación de objetos significantes para los participantes, además de tener sustento en la cual descansa en la integración que los sentidos desarrollan al activar estructuras que participan en la orientación y la atención así como en actividades sensorimotoras (Monsalve-Robayo y Rozo-Reyes, 2009).

Los elementos sensoriales debieran de reflejar la película a proyectarse, tener un significado para las personas y colaborar en la generación de una experiencia emocionalmente grata. Los elementos sensoriales además deberían de ser fáciles de encontrar e incorporar dentro de la misma proyección.

Se dividió a la película en tres partes similares en duración (30 a 35 minutos), esto debido a que las personas mayores con demencia tienen problemas con mantener la atención durante largo tiempo, debido a problemas funcionales y mentales.

A cada división se le asignaron los elementos sensoriales previamente encontrados. Antes de iniciar con las intervenciones se habló con el personal a cargo, se les dio una inducción de 30 minutos sobre el tema de reminiscencia y sobre lo que se buscaba con esta terapia. Los procedimientos utilizados en los participantes se realizaron después de haber entregado las cartas de consentimiento informado a la dirección de la institución. Además, a los participantes, de manera grupal se les aplicó oralmente el Test de Memoria Autobiográfica (AMT) que se retomó de la versión española (Ricarte-Trives et al., 2013), este test consistió en preguntarle al grupo recuerdos relacionados con 10 palabras (positivas y negativas). Lo que se observó fue la casi nula participación grupal, solo una o dos personas respondían y las respuestas eran asociaciones semánticas, lo cual significó que el estado general del grupo podría no ser muy bueno en cuanto a la memoria autobiográfica.

\section{La primera sesión}

Se inició con una introducción sobre lo que se realizaría. Se describió la sesión usando las siguientes palabras: "Hoy vamos a ver una película, una comedia", se preparó material para involucrar a las personas con la película exhibida. Antes de proyectar la película, se inició con estimulación auditiva retomando la banda 
sonora del filme, llamada "Rosalía". Esta canción fue representativa de los años cincuenta en México, podemos encontrarla interpretada por diversos cantantes, entre ellos Pedro Infante. La canción utilizada se retomó del álbum llamado "Valses mexicanos del recuerdo". Con la melodía de fondo se inició la interacción con las personas mayores.

Posteriormente a la inducción sobre lo que se iba tratar la sesión de ese día, se les entregó en mano a cada una de las personas participantes una serie de hojas impresas en papel revolución (papel que asemeja al utilizado en los periódicos, medio impreso emblemático de la generación a tratar), dichas hojas estaban en un orden específico, la primera fue imagen de la cartelera publicada en el periódico: El Sol de Toluca con fecha del 14 de marzo 1954, posteriormente se encontraban imágenes de los actores principales de la película, así como fotografías de las escena. También se encontraba una imagen de unos cigarrillos vendidos en los años cincuenta (de marca: Delicados) así como un pedazo de tela de manta. Los dos últimos elementos se extrajeron de la trama de la película, puesto que al iniciar el filme el diálogo entre los personajes ocurre cuando el actor principal se dirige a un joven vestido con ropa de manta y le entrega un cigarro.

Al exhibirse la película se siguió la trama de ésta, en el momento en el que el personaje principal vendía productos como jabones y perfumes, se hizo entrega a cada uno de los participantes de un jabón envuelto en papel de china de color blanco (de marca: Heno de pravia), de color durazno (jabón de flor de azahar y jabón de avena) y de color negro (jabón maja fragancia original). La envoltura de los jabones se realizó de esta manera debido a que en la década de los años 50's en México, así eran vendidos. Además de los jabones a ciertos participantes se les entregó un puro (habano) por el hecho del papel que este objeto representó tanto como en la película como en la vida cotidiana de los años cincuenta.

\section{La segunda sesión}

Se continuó con 35 minutos más de la película, se hizo estimulación del gusto con la ayuda de la comida proyectada en el filme, en este caso fueron buñuelos. Posteriormente se hizo entrega de papel picado, esto debido a que en la proyección se utiliza dicho recurso para ejemplificar una fiesta patronal.

\section{La tercera sesión}

Se concluyó la película, haciendo uso de bebida espumosa sabor manzana así como de plumas de ave color café. Lo anterior nuevamente retomado de la película, debido a que la bebida alcohólica y las peleas de gallos estuvieron presentes en el filme.

En las tres sesiones se utilizó un lenguaje amistoso, enfocado en captar sensaciones y expresiones de los participantes, todo bajo técnicas etnográficas como la observación participante así como el llevar una bitácora escrita y oral.

No se volvió a aplicar el test AMT, debido a que durante las intervenciones se decidió sólo captar cualitativamente las reacciones emocionales de las personas mayores, los relatos y respuestas a los estímulos, además de que el grupo no se mantuvo en las condiciones iniciales, pues no todos los participantes iniciales concluyeron las sesiones.

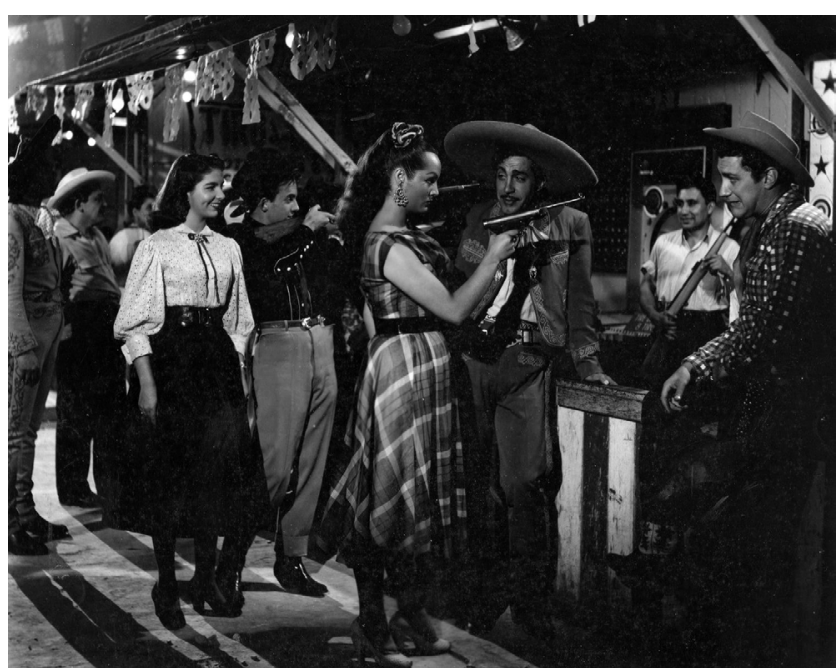

\section{Resultados}

Las personas mayores se mostraron interesadas al hablar de películas y cine. Cabe resaltar que una de las personas con mayores problemas de funcionalidad y con escaso lenguaje se incorporó y abrió los ojos al momento de escuchar la palabra "Coliseo" (que era el nombre de uno de los cines más representativos de la ciudad de Toluca), además de incorporarse, intentó hablar y expresar que ella conoció el cine Coliseo y que recordaba muy bien a la actriz Sara Montiel, quien fue la actriz principal de la película escogida.

El hombre al que se le entregó el puro en la primera sesión, tuvo una reacción emotiva al recibirlo, dijo: 
"Esto es para, para, para...", esta persona se encuentra perdiendo el lenguaje así que se le dijo que, en efecto, el puro era para fumar. Se pudo platicar con la persona haciendo preguntas relacionadas con el puro. La persona asentía con la cabeza y sonreía. La mayoría de las mujeres durante la exhibición de la película estuvieron oliendo los jabones, algo que es de importancia, pues muchas de ellas tienen muy poca movilidad articular.

De manera generalizada, las tres sesiones haciendo uso del cine mexicano de la época de oro fueron exitosas, y este éxito se enuncia debido a que las personas que más deambulan y están más ansiosas se relajaron y sentaron durante las sesiones. El gusto y el olfato fueron los sentidos que más reacciones positivas tuvieron por parte de los participantes.

\section{Discusión y conclusión}

Como hallazgo más significativo se encontró que es posible impartir una terapia sin elementos infantilizantes, sin improvisaciones y que además sea accesible económicamente. El cine mexicano de la época de oro brinda una estructura y otorga pautas a seguir si se analiza previamente lo que se quiere transmitir y lo que se quiere obtener.

El nivel de profesionalización de los cuidadores principales de la gente intervenida, tiene áreas de oportuni- dad pues, aún, se escucharon comentarios negativos en torno a la demencia, comentarios provenientes de los mismos cuidadores. La falta de conocimiento sobre la terapia de reminiscencia y sus alcances fue un obstáculo, que se superó realizando inducciones al personal, aunque aún se debe de trabajar en ello.

Dentro del grupo existían personas con deterioro cognitivo leve, así como gente de 55 años que presentaba algún problema de funcionalidad. Esto se solucionó haciendo uso del cine como eje principal, pero siempre pensando en cada uno de los participantes. El aspecto económico es uno de los limitantes mayores, la solución fue buscar elementos accesibles y replicables.

Futuras investigaciones pueden centrarse en medir el antes y el después de la capacitación y comprensión de la dinámica y ejecución por parte de los cuidadores principales. Así como la memoria autobiográfica, la depresión o ansiedad de los participantes. Los pasos siguientes involucran una mayor cantidad de centros de día o residencias, así como el diseño de un modelo de tratamiento centrado en la emoción y el recuerdo.

Los países que presenciaron la influencia del cine mexicano durante la primera mitad del siglo XX, son candidatos a hacer uso del mismo, sólo se requiere de disposición y pocos recursos materiales para llevarla a cabo.

Financiamiento: El presente documento forma parte de la investigación de carácter doctoral financiada por el CONACYT como parte de las becas a los doctorantes de los posgrados de calidad en México.

Conflicto de intereses: Los autores declaran no tener ningún conflicto de intereses.

Agradecimientos: Se agradece al CONACYT por el apoyo, así como al centro de día, residencia geriátrica y a quienes laboran en el archivo histórico municipal de la ciudad de Toluca por su disposición y colaboración para este proyecto.

\section{Referencias}

Aguirre, J. M. B. (2015). Hay insuficientes geriatras en México, advierte INAPAM. El Universal.Obtenido del sitio: http://archivo. eluniversal.com.mx/nacion-mexico/2015/insuficientes-geriatras-en-mexico-inapam-1083850.html

Baudrillard, J. (2010). El sistema de los objetos. Ciudad de México, México: Siglo XXI.

BBC. (2012). Recuperar los recuerdos con películas antiguas. Obtenido del sitio:http://www.bbc.com/mundo/noticias2012/05/120518_demencia_peliculas_antiguas_m en.shtml

Beard, J., Officer, A., Cassels, A., Bustreo, F., Worning, A. M., y Asamoa-Baah, A. (2015). Informe mundial sobre el envejecimiento y la salud. E.U.A.: OMS

Berntsen, D., y Bohn, A. (2009). Cultural life scripts and individual life stories. En P. Boyer y J. Wertsch (Eds.), Memory in mind and culture: Cambridge.

Buse, C., y Twigg, J. (2014). Women with dementia and their handbags:Negotiating identity, privacy and 'home' through material culture. Journal of Aging Studies, 30, 14-22.

Buse, C., y Twigg, J. (2015). Materialising memories: Exploring the stories of people with dementia through dress. Ageing and Society. doi:10.1017/S0144686X15000185 
Castro-Ricalde, M. (2014). El cine mexicano de la edad de oro y su impacto internacional. La Colmena, 82, 9-16.

Castro-Ricalde, M., y Irwin, R. M. (2011). El cine mexicano "se impone” mercados internacionales y penetración cultural en la época dorada. México DF: UNAM.

Cotelli, M., Manenti, R., y Orazio, Z. (2012). Reminiscence therapy in dementia: A review. Maturitas, 72(2012), 203-205. doi: http:// dx.doi.org/10.1016/j.maturitas.2012.04.008

Dementia Village Architects.(2015). Villas para la demencia. Obtenido de: http://dementiavillage.com

Dulcey-Ruiz, E. (2015). Envejecimiento y vejez. Categorías y conceptos R. L. d. G.-S. d. H. Editores (Ed.) .

García-Riera, E. (1974). El cine y su público. México DF: Fondo de Cultura Económica.

Geertz, C. (2006). La interpretación de las culturas (Undécima ed.). México: Gedisa.

Iacub, R. (2011). Identidad y envejecimiento. Buenos Aires: Paidós.

Im still here foundation. (2016). Meet me at the movies... and make memories. Obtenido de: http://www.imstillhere.org/takes-a-village/it-takes-village/movies

INEGI. (2010). Censo de población y vivienda. México. Obtenido de: http://www.beta.inegi.org.mx/proyectos/ccpv/2010/

Kalu, M. (2015). Literature review on the effectiveness of using reminiscence therapy in treating dementia.O btenido de: https:// www.researchgate.net/publication/275656274_Literature_review_on_the_effectiveness_of_using_Reminiscence_therapy_in_treating_Dementia\%28_only_4_Articles\%29 doi:10.13140/RG.2.1.4105.8720

Latha, K. S., Bhandary, P. V., Tejaswini, S., y Sahana, M. (2014). Reminiscence therapy: An overview. Middle East Journal of Age and Ageing, 11(1).

Malafouris, L., y Renfrew, C. (2013). How Things Shape the Mind: A Theory of Material Engagement. MIT Press.

Martínez-Rodríguez, T. (2011). La atención gerontológica centrada en la persona: Guía para la intervención profesional en los centros $y$ servicios de atención a personas mayores en situación de fragilidad o dependencia. E. J. A. Z. Nagusia (Ed.)

Memory-bank. (2012). Memory bank. Obtenido de: https://www.rcpsych.ac.uk/pdf/Memory\%20Bank\%20-\%20Linda\%20 Brown.pdf

Monsalve-Robayo, A. M., y Rozo-Reyes, C. M. (2009). Integración sensorial y demencia tipo alzheimer principios y métodos para la rehabilitación. Rev. Colombiana de Psiaquiatría, 38(4). OMS. (2015). Demencia. En OMS (Ed.), Nota descriptiva No. 362

Prince, M., Wimo, A., Guerchet, M. A., Gemma-Claire., Wu., Y.-T., y Prina, M. (2015). The global impact of dementia. Obtenido de: http://www.alz.co.uk/research/WorldAlzheimerReport2015.pdf

Ricarte-Trives, J. J., Latorre-Postigo, J. M., y Ros-Segura, L. (2013). Diseño y análisis del funcionamiento del test de memoria autobiográfica en población española. Apuntes de Psicología, 31(1), 3-10.

Sosa, A. L. (2016). XXI Congreso Nacional de Alzheimer. León Guanajuato México.

Tulving, E. (1972). Episodic and semantic memory. En E. Tulving y W. Donaldson (Eds.), Organization of memory. New York: Academic Press.

Williams, H. L., y Conway, M. (2009). Networks of autobiographical memories. En P. Boyer y J. Wertsch (Eds.), Memory in mind and culture: Cambridge. 